\title{
Características de los componentes del síndrome metabólico en pacientes con glucosa normal y disglicemia en ayunas
}

\author{
Characteristics of the components of the metabolic syndrome in patients with normal glucose and fasting \\ disglicemia
}

Fernando de Jesús Gross Melo ${ }^{1,2, a, e}$, Juan Jorge Huamán Saavedra ${ }^{2, c, f}$, Jaime Gonzáles Saldaña ${ }^{2, d}$, Jessica Gutiérrez Arana $^{2, d}$, Mayita Álvarez Vargas ${ }^{2, d}$, Jorge Enrique Osada Liy 1,b

\section{RESUMEN}

Objetivos: Investigar las características de los componentes del Síndrome Metabólico (SM) entre pacientes con glucosa normal y disglicemia en ayunas en la población adulta de Trujillo. Material y métodos: Estudio de análisis secundario tipo descriptivo, comparativo, relacional. Se analizó base de datos, de un estudio previo sobre SM en adultos de 20-79 años en Trujillo entre los años 2010 al 2014, desarrollado a través de campañas médicas de despistaje de factores de riesgo cardiovascular. El grupo 1: incluyó 127 adultos con SM y glucosa normal en ayunas (NG). El grupo 2: incluyó 110 adultos con SM y disglicemia en ayunas (DG). Se usó la prueba t de Student para comparación de medias y la prueba $\mathrm{Z}$ para comparación de proporciones, con un nivel de significancia de 0,05 e intervalos de confianza del 95\%. Resultados: Los niveles séricos de triglicéridos en ayunas (TG) fueron 219,09 \pm $101,83 \mathrm{mg} / \mathrm{dl}$ en NG y $192,3 \pm 103,17 \mathrm{mg} / \mathrm{dl}$ en DG ( $\mathrm{p}=0,004)$ y la frecuencia de hipertrigliceridemia fue $85,04 \%$ en NG y $63,64 \%$ en DG ( $p=0,000)$ y de HDL colesterol bajo $71,65 \%$ en NG y $51,81 \%$ en DG $(p=0,002)$. Conclusiones: Existe mayor nivel sérico de TG y mayor frecuencia de hipertrigliceridemia y HDL colesterol bajo en el grupo NG.

PALABRAS CLAVE: Síndrome X Metabólico, glucosa, adulto. (Fuente: DeCS BIREME).

\section{SUMMARY}

Objectives: To investigate the characteristics of the components of metabolic syndrome (MS) among patients with normal glucose and fasting dysglycemia in the adult population of Trujillo. Methods: Secondary analysis of descriptive, comparative and relational type. Database was analyzed of MS previous study in adults of 20-79 years in Trujillo between 2010 to 2014, developed through medical screening campaigns cardiovascular risk factors. Group 1: MS included 127 adults with normal fasting glucose (NG). The group 2 included 110 adults with MS and dysglycemia fasting (DG). Student $t$ test was used to compare means and the $\mathrm{Z}$ test to compare proportions, with a significance level of 0.05 and confidence intervals of $95 \%$. Results: Serum levels of fasting triglyceride (TG) were $(219.09 \pm 101.83 \mathrm{mg} / \mathrm{dl}$ and $192.3 \pm \mathrm{NG} 103.17 \mathrm{mg} / \mathrm{dl}$ in DG, $\mathrm{p}=0.004)$ and frequency of hypertriglyceridemia was $(85.04 \%$ and $63.64 \%$ in NG in DG with $\mathrm{p}=0.000)$ and low HDL cholesterol $(71.65 \%$ and $51.81 \%$ in NG in

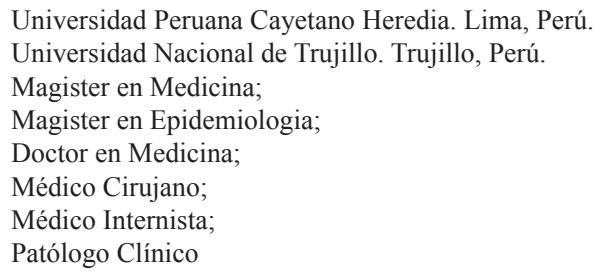


DG, $\mathrm{p}=0.002)$. Conclusions: Higher level of TG and increased frequency of hypertriglyceridemia and low HDL cholesterol in the NG group.

KEYWORDS: Metabolic Syndrome X, glucose, adult. (Source: MeSH NLM).

\section{INTRODUCCIÓN}

El Síndrome Metabólico (SM) es un conjunto de factores de riesgo para diabetes mellitus tipo 2 y enfermedad cardiovascular, caracterizado por resistencia a la insulina (RI) o adiposidad de predominio visceral, asociados con trastorno del metabolismo de los carbohidratos y lípidos, y cifras elevadas de presión arterial (1-3).

En Perú la prevalencia varía entre 16 y $28 \%(4,5)$, al igual que en Trujillo (6).

Dentro de su polimorfismo y heterogeneidad se ha encontrado que el SM está muy asociado y tiene una patogenia en muy estrecha relación con la prediabetes y la diabetes, por ello la Organización Mundial de la Salud (OMS), la European Group for Study of Insulin Resistency (EGIR), y la American Association of Clinical Endocrinologist (AACE), consideran que la glucosa alterada en ayunas, la intolerancia a la glucosa, la diabetes mellitus tipo 2 o la RI constituyen un criterio diagnóstico prioritario del SM $(7,8)$.

La prevalencia de cada uno de los componentes del SM puede variar y en el caso de la hiperglicemia está entre 24 y $100 \%$ (9), además ser el componente menos prevalente $(5,6,9)$. En Perú, la prevalencia de hiperglicemia en pacientes con SM es variable, $8 \%$ en promedio (10) y en Trujillo, Galarreta la encontró entre $40-43 \%$ (6).

En Trujillo, en el 2011, Castillo et al (11), en un estudio poblacional en adultos, encontraron que, con el aumento de la glucosa basal alterada, se incrementaron gradualmente: cintura abdominal, el índice de masa corporal, las presiones sistólica y diastólica y disminuyó el HDL colesterol (HDL col).

Estudiando la influencia de la glicemia, en Japón, se sometió a sujetos normoglicémicos a un test de tolerancia y separándolos por cuartiles, se encontró que a más niveles de glucosa mayor correlación con obesidad, hipertensión e hiperlipidemia (12). En Taiwán, en adultos normoglicémicos mayores de 65 años, agrupados en 3 niveles de glicemia $(<90,90-$ 95 y $95-100 \mathrm{mg} / \mathrm{dl}$ ), se evidenció que los adultos con glicemia $>90 \mathrm{mg} / \mathrm{dl}$ tenían mayor porcentaje de grasa corporal, mayores niveles séricos de LDL colesterol (LDL) y triglicéridos y menores niveles de HDL col (13).

En Turquía, Uzunlulu et al (14), concluyeron que la hiperglicemia y el SM, eran conceptos independientes al encontrar 6,5\% de sujetos normoglicémicos sin SM que eran intolerantes a la glucosa, y que las cifras de presión arterial, cintura abdominal, y nivel de triglicéridos eran semejantes entre sujetos con SM con glucosa normal y disglicemia.

Tomando en cuenta la importancia del SM, la variación de su frecuencia en los diversos países y de sus componentes, con una prevalencia en general menor de hiperglicemia, así como, la influencia que podría ejercer la glucosa alterada sobre el resto del perfil metabólico, el trabajo tuvo como objetivo investigar las características de los componentes del síndrome metabólico entre pacientes con glucosa normal y disglicemia en adultos de Trujillo, Perú.

\section{MATERIAL Y MÉTODOS}

Estudio de análisis secundario de tipo descriptivo, comparativo, relacional de información de base de datos de trabajo previo sobre SM desarrollado en Trujillo, en adultos de raza mestiza de 20-79 años entre los años 2010 al 2014, a través de campañas médicas de despistaje de factores de riesgo cardiovascular, previa difusión por volanteo en cada zona (15).

El estudio utilizó para el análisis y selección de la muestra al total de población incluida. De un total de 2164 personas voluntarias, solo 1364 aceptaron participar en el estudio, lográndose incluir en total a 639 adultos (301 varones y 338 mujeres).

Del total de la población se hizo una selección al azar, en forma aleatoria simple entre los que cumplían los criterios de selección, hasta complementar los grupos por edad y género. Se logró reunir, 237 adultos con SM. Para determinar si esta muestra era significativa, se aplicó la potencia de la prueba para estudio comparativo de medias, lográndose conseguir una potencia de prueba de $84,65 \%$, necesaria para 
un estudio comparativo de medias (de acuerdo a la diferencia en HDL encontrada en (14)).

Muestra: 237 adultos con SM (112 varones y 125 mujeres), de los cuales 127 tuvieron glucosa normal y 110 disglicemia en ayunas.

Criterios de Inclusión: los mismos del estudio original. Adultos de 20 a 79 años con SM, clasificados según definición de consenso JIS, que incluye a personas con glucosa mayor de $100 \mathrm{mg} / \mathrm{dl}$, prediabéticos y diabéticos con o sin tratamiento.

Criterios de exclusión: los mismos del estudio original. Gestantes, diabéticos tipo 1, personas usuarias de fármacos hipo o hiperlipemiantes y personas con enfermedad aguda o crónica sistémica que pudieran alterar los valores antropométricos o de laboratorio o que rechazaron participar en el estudio.

\section{Procedimiento y definición operacional}

En el grupo 1 se incluyeron pacientes adultos entre 20 y 79 años con SM y glucosa normal en ayunas
(NG); en el grupo 2, se incluyeron pacientes adultos entre 20 y 79 años con SM y disglicemia en ayunas (DG).

SM según JIS (SM JIS): Definición armonizante, presencia de, al menos, tres de los siguientes factores de riesgo: obesidad abdominal (circunferencia de la cintura en hombre $\geq 90 \mathrm{~cm}$ y en mujeres $\geq 80 \mathrm{~cm}$ ); triglicéridos $(\mathrm{TG}) \geq 150 \mathrm{mg} / \mathrm{dl} ; \mathrm{HDL}$ col $<40 \mathrm{mg} / \mathrm{dl}$ en varones y $<50 \mathrm{mg} / \mathrm{dl}$ en mujeres; presión arterial $\geq 130 / 85 \mathrm{~mm} \mathrm{Hg}$, historia de hipertensión arterial y/o tratamiento farmacológico; glucosa en ayunas $\geq 100 \mathrm{mg} / \mathrm{dl}$, diagnóstico de DM 2 y/o tratamiento farmacológico (2).

Glucosa normal en ayunas (NG): Pacientes con muestra sanguínea de glucosa luego de un ayuno nocturno de 10-12 horas, con valor menor de 100mg/ $\mathrm{dl}(16)$.

Disglicemia en ayunas (DG): Pacientes con muestra sanguínea de glucosa, luego de un ayuno nocturno de 10-12 horas, con valor mayor o igual de $100 \mathrm{mg} / \mathrm{dl}$ (16) y por estudios previos semejantes también se incluyó

Tabla 1. Comparación de los componentes del Síndrome Metabólico en Trujillo, años 2010-2014, según glicemia.

\begin{tabular}{lccc}
\hline & $\begin{array}{c}\text { NORMOGLICEMIA } \\
(\mathbf{n}=\mathbf{1 2 7}) \\
(\text { Media } \pm \mathbf{D E})\end{array}$ & $\begin{array}{c}\text { DISGLICEMIA } \\
(\mathbf{n}=\mathbf{1 1 0}) \\
(\text { Media } \pm \mathbf{D E})\end{array}$ & $\mathbf{p}$ \\
\hline Edad & $53,98 \pm 14,45$ & $57,91 \pm 12,79$ & 0,032 \\
IMC (kg/m $)$ & $28,17 \pm 3,85$ & $28,56 \pm 4,46$ & 0,79 \\
Cintura (cm) & $93,74 \pm 9,72$ & $94,75 \pm 11,43$ & 0,66 \\
PAS (mm Hg) & $126,76 \pm 14,38$ & $127,88 \pm 14,42$ & 0,42 \\
PAD (mm Hg) & $79,20 \pm 9,07$ & $78,36 \pm 8,35$ & 0,57 \\
Triglicéridos (mg/d) & $219,09 \pm 101,83$ & $192,3 \pm 103,17$ & 0,004 \\
Colesterol total (mg/d) & $227,52 \pm 43,30$ & $227,52 \pm 51,35$ & 0,53 \\
LDL (mg/dl) & $139,46 \pm 44,22$ & $144,2 \pm 48,93$ & 0,82 \\
HDL (mg/dl) & $43,49 \pm 7,65$ & $44,92 \pm 7,86$ & 0,19 \\
Glicemia (mg/dl) & $87,75 \pm 6,79$ & $134,95 \pm 50,25$ & 0,000 \\
No de criterios de SM & $3,21 \pm 0,40$ & $3,75 \pm 0,70$ & 0,000 \\
Factor TG/HDL (n\%) & $109(85,8)$ & $80(72,7)$ & 0,013 \\
TG $>$ 130 (n\%) & $114(89,8)$ & $87(79,1)$ & 0,023 \\
\hline
\end{tabular}

$\mathrm{IMC}=$ índice de masa muscular, $\mathrm{PAS}=$ presión arterial sistólica, $\mathrm{PAD}=$ presión arterial diastólica, $\mathrm{LDL}=\mathrm{LDL}$ colesterol, $\mathrm{HDL}=\mathrm{HDL}$ colesterol, $\mathrm{TG}=$ triglicéridos / nivel de significancia $\mathrm{p}$, según prueba $\mathrm{T}$ para muestras independientes para componentes 1-11 y prueba $\mathrm{Z}$ para componentes 12 y13 
en este grupo a pacientes diabéticos o en tratamiento farmacológico $(14,17)$.

\section{Mediciones y técnicas}

Se tuvo acceso a la base de datos completa de 639 personas, la misma que no contaba con información que permitiera identificar a los participantes y se tomaron los datos únicamente de los individuos con SM con glucosa normal y disglicemia.

Las mediciones estandarizadas de los componentes del SM se realizaron de acuerdo a guías ya establecidas y aceptadas y fueron reportadas en el estudio original (15) (peso, talla, índice de masa muscular (IMC), circunferencia abdominal (CA), presión arterial), así como la toma de muestras sanguíneas fue en condiciones de 10 a $12 \mathrm{~h}$ de ayuno con métodos conocidos.

\section{Plan de Análisis}

Los datos recolectados fueron procesados mediante tablas en Microsoft Excel 2010 y el análisis estadístico se llevó a cabo en el paquete estadístico IBM SPSS ${ }^{\circledR}$ v22.0.

Primero, se evaluaron las características poblacionales del total de sujetos con SM, seguidamente se realizó la comparación entre los grupos, con glucosa normal y disglicemia, de las variables presión arterial sistólica, presión arterial diastólica, CA, IMC, triglicéridos (TG), HDL colesterol, considerando además la edad y el sexo. La normalidad de las variables se evaluó con la prueba Kolmogórow-Smirnow, se usó la prueba t de Student para muestras independientes (variables paramétricas) y la prueba $\mathrm{Z}$ para comparación de proporciones (18). Los resultados fueron evaluados a un nivel de significancia de 0,05 con intervalos de confianza al $95 \%$.
El estudio fue exonerado de revisión por el Comité de Ética de la Universidad Peruana Cayetano Heredia.

\section{RESULTADOS}

Los niveles séricos de triglicéridos en ayunas (TG) fueron $219,09 \pm 101,83 \mathrm{mg} / \mathrm{dl}$ en NG y 192,3 $\pm 103,17$ $\mathrm{mg} / \mathrm{dl}$ en $\mathrm{DG}(\mathrm{p}=0,004)($ Tabla 1$)$ y la frecuencia de hipertrigliceridemia fue $85,04 \%$ en NG y $63,64 \%$ en DG $(\mathrm{p}=0,000)$ y de HDL colesterol bajo fue $71,65 \%$ en NG y $51,81 \%$ en DG $(p=0,002)$ (Tabla 2$)$.

Respecto a la distribución de la población, de los 237 participantes pareados por edad y sexo, el $52,74 \%$ correspondió al sexo femenino, distribución con preponderancia femenina que se mantuvo en los grupos etarios de 40 a 59 años y de 60 a 79 años. Siendo la edad promedio de la muestra 55,81 $\pm 13,81$ (Tabla 3).

En el grupo de individuos disglicémicos, el 20\% eran diabéticos confirmados o estaban en tratamiento farmacológico, 23\% tenían glucosa en ayunas > 126 $\mathrm{mg} / \mathrm{dl}$ (posiblemente diabéticos) y el 57\% tenían glucosa alterada en ayunas $>100 \mathrm{mg} / \mathrm{dl}$.

En los gráficos 1 y 2 se muestra la frecuencia de cada uno de los componentes del SM de acuerdo al sexo y la glicemia manteniéndose la mayor frecuencia de hipertrigliceridemia y de HDL col bajo en los hombres normoglicémicos.

\section{DISCUSIÓN}

Evaluando la tabla 1, encontramos que los individuos del grupo con DG tienen mayor edad, menor nivel sérico de TG, mayor glicemia y mayor numero promedio de componentes del SM en forma significativa. La diferencia en el nivel de TG, se mantiene en forma semejante cuando se los separa por sexo, al igual que por grupos etarios, con pequeñas

Tabla 2. Frecuencia de los componentes individuales del síndrome Metabólico en Trujillo-años 2010-2014, en forma global y según glicemia.

\begin{tabular}{lcccc}
\hline COMPONENTE & $\begin{array}{c}\text { TOTAL } \\
(\mathbf{N}=\mathbf{2 3 7})\end{array}$ & $\begin{array}{c}\text { Normoglicemia } \\
(\mathbf{N}=\mathbf{1 2 7})\end{array}$ & $\begin{array}{c}\text { Disglicemia } \\
(\mathbf{N}=\mathbf{1 1 0})\end{array}$ & P \\
\hline Cintura & $224(94,50 \%)$ & $123(96,85 \%)$ & $101(91,81 \%)$ & 0,098 \\
Hipertensión & $161(67,90 \%)$ & $86(67,72 \%)$ & $75(68,18 \%)$ & 0,939 \\
hipertrigliceridemia & $178(75,10 \%)$ & $108(85,04 \%)$ & $70(63,64 \%)$ & 0,000 \\
HDL bajo & $148(62,44 \%)$ & $91(71,65 \%)$ & $57(51,81 \%)$ & 0,002 \\
\hline
\end{tabular}

p: significancia estadística según prueba $\mathrm{z}$ 


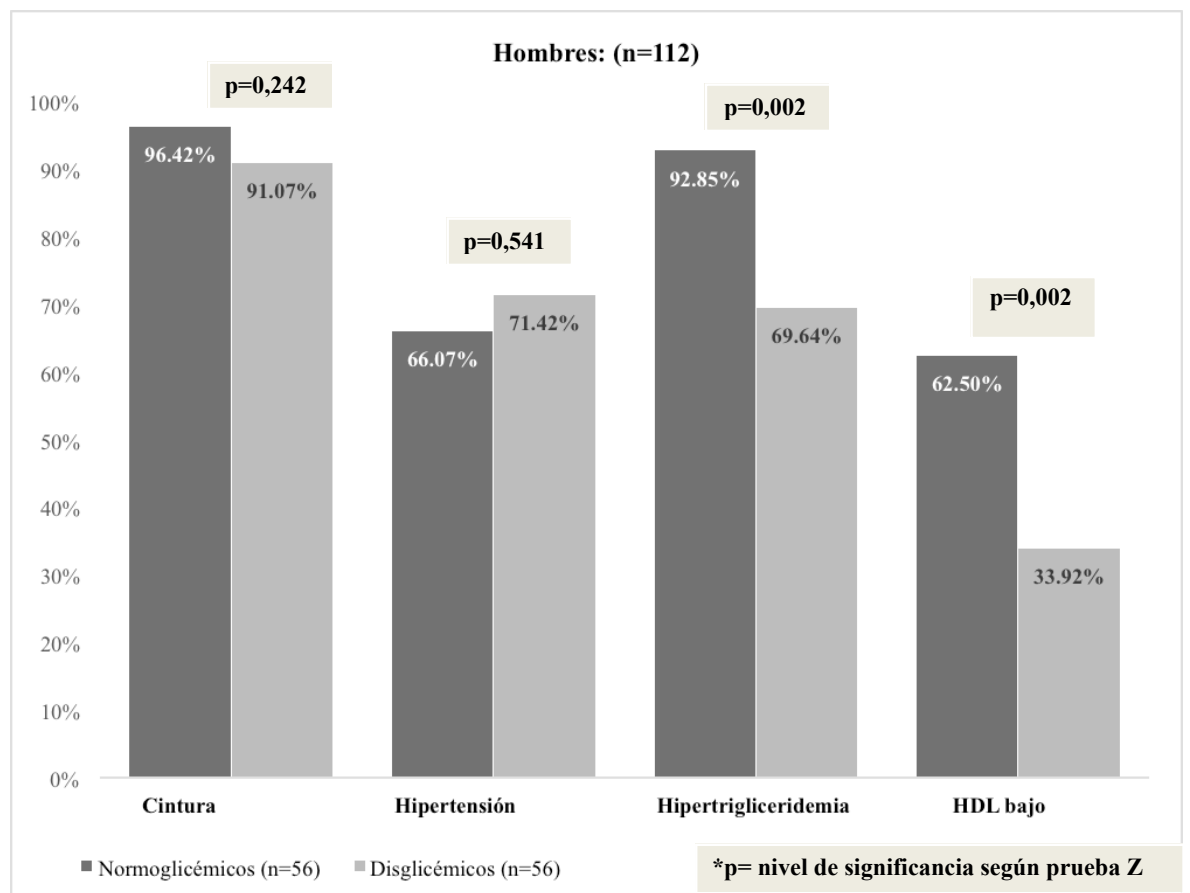

Gráfico 1. Frecuencia de los componentes del síndrome metabólico en Trujillo -años 2010-2014-según sexo masculino.

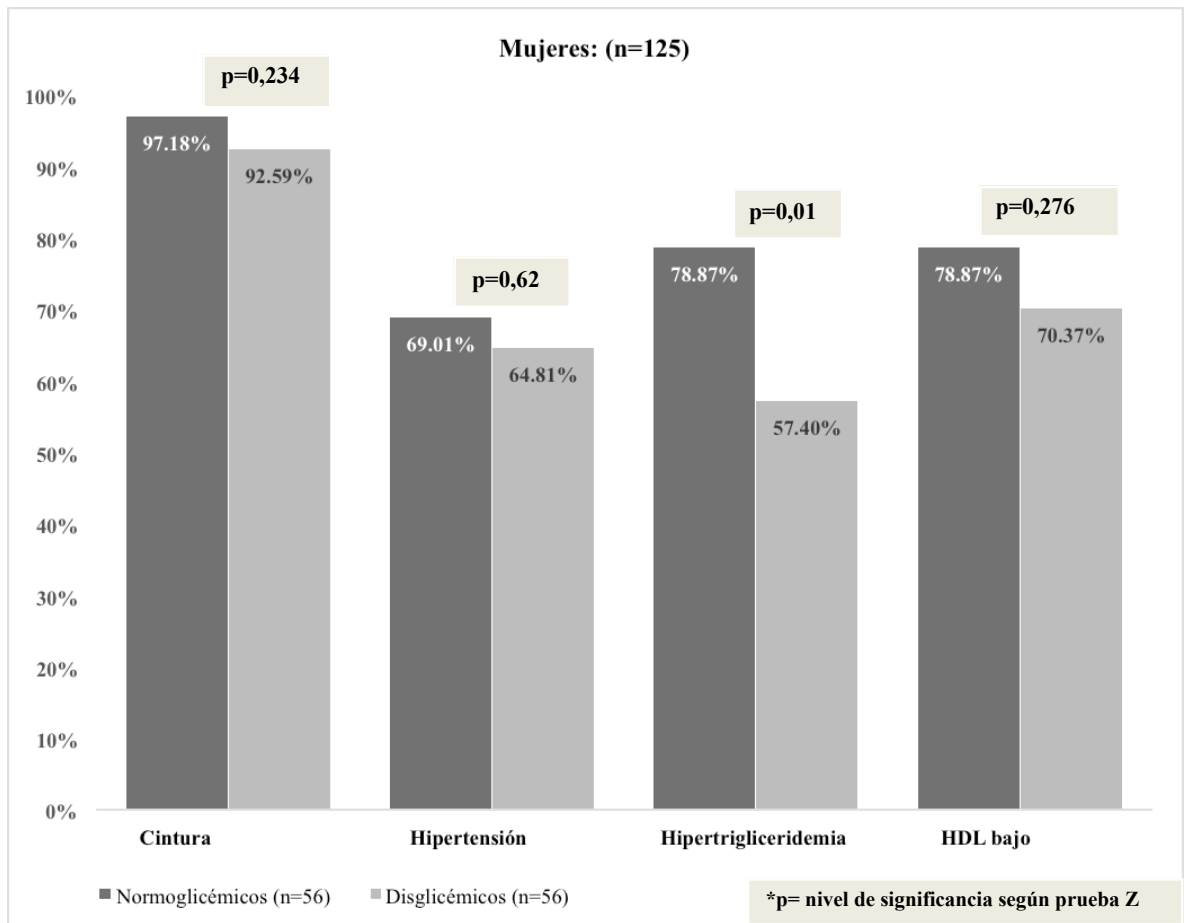

Gráfico 2. Frecuencia de los componentes del síndrome metabólico en Trujillo -años 2010-2014-según sexo femenino.

diferencias en algunos grupos etarios masculinos no relevantes para el total (Tabla 4).

Nuestros hallazgos se comparan con el estudio Turco (14) donde se analizó primero las diferencias de los componentes sin considerar si tenían SM; la presión arterial, CA y el nivel sérico de TG resultaron en mayor niveles estadísticamente significativos y menor HDL col para el caso de los DG, incluso estos resultados se mantuvieron cuando se separó a los DG en intolerantes a la glucosa y diabéticos; pero cuando se buscaron las diferencias entre NG y DG con SM, 
Tabla 3. Frecuencia de disglicemia en los pacientes con síndrome metabólico en Trujillo, años 2010-2014, según edad y sexo.

\begin{tabular}{lcccccc}
\hline & \multicolumn{2}{c}{$\begin{array}{c}\text { Disglicemia } \\
(\mathbf{N}=\mathbf{1 1 0})\end{array}$} & \multicolumn{2}{c}{$\begin{array}{c}\text { Normoglicemia } \\
(\mathbf{N}=\mathbf{1 2 7})\end{array}$} & \multicolumn{2}{c}{$\begin{array}{c}\text { Total } \\
(\mathbf{N}=\mathbf{2 3 7})\end{array}$} \\
\cline { 2 - 7 } & $\mathbf{n}$ & $\mathbf{\%}$ & $\mathbf{n}$ & $\mathbf{\%}$ & $\mathbf{n}$ & $\mathbf{\%}$ \\
\hline VARONES & & & & & & \\
$\mathbf{2 0}$ a 39 años & 9 & 16,1 & 16 & 28,6 & 25 & 22,3 \\
$\mathbf{4 0}$ a 59 años & 19 & 33,9 & 27 & 48,2 & 46 & 41,1 \\
60 a 79 años & 28 & 50,0 & 13 & 23,2 & 41 & 36,6 \\
Total & 56 & 50,9 & 56 & 44,1 & 112 & 47,3 \\
MUJERES & & & & & & \\
20 a 39 años & 2 & 3,7 & 7 & 9,9 & 9 & 7,2 \\
40 a 59 años & 25 & 46,3 & 28 & 39,4 & 53 & 42,4 \\
60 a 79 años & 27 & 50,0 & 36 & 50,7 & 63 & 50,4 \\
Total & 54 & 49,1 & 71 & 55,9 & 125 & 52,7 \\
\hline
\end{tabular}

Valor-p según prueba Chi Cuadrado. $\mathrm{p}=0,295$ para diferencia entre seso y $\mathrm{p}=0,10$ para edad.

Tabla 4. Componentes del Síndrome Metabólico en los pacientes con glucosa normal y disglicemia según género y grupos etarios, Trujillo $2010-2014$.

\begin{tabular}{|c|c|c|c|c|c|c|c|c|c|c|}
\hline & \multirow[b]{2}{*}{ EDAD } & \multicolumn{2}{|c|}{ VARONES } & \multirow[b]{2}{*}{$\mathbf{p}$} & \multicolumn{2}{|c|}{ MUJERES } & \multirow[b]{2}{*}{$\mathbf{p}$} & \multicolumn{2}{|c|}{ TOTAL } & \multirow[b]{2}{*}{$\mathbf{p}$} \\
\hline & & $\begin{array}{l}\text { GLICEMIA } \\
\text { NORMAL }\end{array}$ & DIS GLICEMIA & & $\begin{array}{l}\text { GLICEMIA } \\
\text { NORMAL }\end{array}$ & DIS GLICEMIA & & $\begin{array}{l}\text { GLICEMIA } \\
\text { NORMAL }\end{array}$ & DIS GLICEMIA & \\
\hline \multirow[t]{4}{*}{ Cintura (cm) } & $20-39$ & $104,0 \pm 10,24$ & $105,0 \pm 17,28$ & NS & $87,14 \pm 5,98$ & $88,5 \pm 26,16$ & NS & $98,87 \pm 12,01$ & $102,0 \pm 18,76$ & NS \\
\hline & $40-59$ & $96,44 \pm 6,09$ & $100,79 \pm 11,55$ & NS & $89,96 \pm 9,48$ & $87,56 \pm 8,83$ & NS & $93,15 \pm 8,57$ & $93,27 \pm 11,97$ & NS \\
\hline & $60-79$ & $102,0 \pm 6,24$ & $97,32 \pm 6,94$ & $<0,05$ & $88,39 \pm 7,12$ & $91,56 \pm 9,04$ & NS & $92,0 \pm 9,14$ & $94,49 \pm 8,48$ & NS \\
\hline & Total & $99,89 \pm 8,15$ & $99,73 \pm 10,89$ & NS & $88,89 \pm 8$ & $89,59 \pm 9,61$ & NS & $93,74 \pm 9,73$ & $94,75 \pm 11,43$ & NS \\
\hline \multirow[t]{4}{*}{ PAS (mm Hg) } & $20-39$ & $123,06 \pm 13,95$ & $122,0 \pm 8,93$ & NS & $113,43 \pm 17,15$ & $105,0 \pm 7,07$ & NS & $120,13 \pm 15,28$ & $118,91 \pm 10,77$ & NS \\
\hline & $40-59$ & $123,89 \pm 16,13$ & $125,26 \pm 10,6$ & NS & $123,5 \pm 12,88$ & $120,88 \pm 16,4$ & NS & $123,69 \pm 14,43$ & $122,77 \pm 14,21$ & NS \\
\hline & $60-79$ & $132,31 \pm 12,52$ & $133,86 \pm 12$ & NS & $133,69 \pm 10,87$ & $133,67 \pm 13,88$ & NS & $133,33 \pm 11,21$ & $133,76 \pm 12,84$ & NS \\
\hline & Total & $125,61 \pm 14,98$ & $129,04 \pm 12$ & NS & $127,68 \pm 13,94$ & $126,69 \pm 16,61$ & NS & $126,76 \pm 14,38$ & $127,88 \pm 14,43$ & NS \\
\hline \multirow[t]{4}{*}{ PAD (mm Hg) } & $20-39$ & $78,75 \pm 9,75$ & $77,78 \pm 6,67$ & NS & $75,71 \pm 10,97$ & $70,0 \pm 14,14$ & NS & $77,83 \pm 9,98$ & $76,36 \pm 8,09$ & NS \\
\hline & $40-59$ & $82,41 \pm 9,44$ & $80,79 \pm 7,12$ & NS & $80,29 \pm 8,08$ & $77,6 \pm 11,28$ & NS & $81,33 \pm 8,76$ & $78,98 \pm 9,74$ & NS \\
\hline & $60-79$ & $76,15 \pm 8,93$ & $79,11 \pm 6,24$ & NS & $77,92 \pm 8,65$ & $77,41 \pm 8,13$ & NS & $77,45 \pm 8,67$ & $78,27 \pm 7,21$ & NS \\
\hline & Total & $79,91 \pm 9,61$ & $79,46 \pm 6,58$ & NS & $78,63 \pm 8,67$ & $77,22 \pm 9,79$ & NS & $79,2 \pm 9,08$ & $78,36 \pm 8,35$ & NS \\
\hline \multirow[t]{4}{*}{ Triglicéridos (mg/dl) } & $20-39$ & $312,0 \pm 123,27$ & $208,67 \pm 63,39$ & $<0,05$ & $198,43 \pm 66,55$ & $169,5 \pm 3,54$ & NS & $277,43 \pm 120,1$ & $201,55 \pm 58,88$ & NS \\
\hline & $40-59$ & $238,85 \pm 87,89$ & $192,89 \pm 89,78$ & NS & $209,79 \pm 114,55$ & $203,76 \pm 94,36$ & NS & $224,05 \pm 102,45$ & $199,07 \pm 91,51$ & NS \\
\hline & $60-79$ & $200,0 \pm 90,54$ & $212,68 \pm 151,17$ & $<0,05$ & $181,14 \pm 73,29$ & $156,37 \pm 61,72$ & NS & $186,14 \pm 77,7$ & $185,04 \pm 118,61$ & NS \\
\hline & Total & $250,73 \pm 106,56$ & $205,32 \pm 120,52$ & NS & $194,14 \pm 91,18$ & $178,8 \pm 80,34$ & NS & $219,09 \pm 101,84$ & $192,3 \pm 103,17$ & 0,004 \\
\hline \multirow[t]{4}{*}{ HDL (mg/dl) } & $20-39$ & $42,25 \pm 9,07$ & $40,56 \pm 5,94$ & NS & $43,29 \pm 4,96$ & $43,5 \pm 7,78$ & NS & $42,57 \pm 7,94$ & $41,09 \pm 5,97$ & NS \\
\hline & $40-59$ & $40,78 \pm 9,03$ & $42,32 \pm 7,97$ & NS & $44,11 \pm 6,23$ & $47,16 \pm 7,51$ & NS & $42,47 \pm 7,84$ & $45,07 \pm 8$ & NS \\
\hline & $60-79$ & $39,38 \pm 4,37$ & $45,75 \pm 7,44$ & $<0,05$ & $47,11 \pm 6,94$ & $45,37 \pm 8,66$ & NS & $45,06 \pm 7,19$ & $45,56 \pm 7,99$ & NS \\
\hline & Total & $40,88 \pm 8,14$ & $43,75 \pm 7,58$ & NS & $45,55 \pm 6,62$ & $46,13 \pm 8,03$ & NS & $43,49 \pm 7,66$ & $44,92 \pm 7,86$ & NS \\
\hline \multirow[t]{4}{*}{ Colesterol total $(\mathrm{mg} / \mathrm{dl})$} & $20-39$ & $227,5 \pm 22,25$ & $209,89 \pm 41,56$ & NS & $212,14 \pm 31,74$ & $272,0 \pm 52,33$ & NS & $222,83 \pm 25,78$ & $221,18 \pm 47,82$ & NS \\
\hline & $40-59$ & $217,7 \pm 58,95$ & $211,05 \pm 49,09$ & NS & $232,04 \pm 37,91$ & $239,96 \pm 42,63$ & NS & $225,0 \pm 49,43$ & $227,48 \pm 47,25$ & NS \\
\hline & $60-79$ & $206,62 \pm 27,39$ & $221,61 \pm 46,07$ & NS & $241,92 \pm 43,67$ & $236,3 \pm 64,4$ & NS & $232,55 \pm 42,73$ & $228,82 \pm 55,79$ & NS \\
\hline & Total & $217,93 \pm 44,7$ & $216,14 \pm 45,96$ & NS & $235,08 \pm 40,92$ & $239,31 \pm 54,35$ & NS & $227,52 \pm 43,31$ & $227,52 \pm 51,36$ & NS \\
\hline \multirow[t]{4}{*}{ LDL (mg/dl) } & $20-39$ & $122,94 \pm 33,44$ & $127,67 \pm 49,15$ & NS & $129,0 \pm 23,63$ & $195,0 \pm 60,81$ & $<0,05$ & $124,78 \pm 30,38$ & $139,91 \pm 55,17$ & NS \\
\hline & $40-59$ & $125,52 \pm 56,66$ & $130,16 \pm 43,71$ & NS & $146,0 \pm 41,99$ & $152,12 \pm 34,85$ & NS & $135,95 \pm 50,34$ & $142,64 \pm 39,98$ & NS \\
\hline & $60-79$ & $127,38 \pm 31,2$ & $133,46 \pm 34,55$ & NS & $158,58 \pm 40,35$ & $159,63 \pm 67,83$ & NS & $150,31 \pm 40,3$ & $146,31 \pm 54,65$ & NS \\
\hline & Total & $125,21 \pm 45,14$ & $131,41 \pm 39,59$ & NS & $150,7 \pm 40,37$ & $157,46 \pm 54,27$ & NS & $139,46 \pm 44,23$ & $144,2 \pm 48,93$ & NS \\
\hline
\end{tabular}


los grupos fueron semejantes y solo se diferenciaron en el nivel de HDL col; y aunque la diferencia del nivel de TG no resultó significativa $(\mathrm{p}=0,08)$, pero numéricamente el nivel plasmático fue mayor en los NG. Las diferencias en HDL col no significativa en nuestro caso, quizá se deban a la distribución asimétrica en los tamaños de muestra entre NG y DG (153 NG en Turquía con 487 DG y 127 NG y 110 DG en nuestra muestra). Además, en nuestro grupo de DG no se separó a los intolerantes a la glucosa, de los pre diabéticos y diabéticos.

De acuerdo a la frecuencia relativa de cada componente del SM en cada grupo, se encontró, en general, que el patrón que diferencia a los NG y DG son las mayores frecuencias de TG y HDL col bajo en los NG (Tabla 2), incluso separada por sexo (Gráficos 1 y 2 ).

Se ha reportado que el SM en el hombre tiene como componente prevalente la hipertrigliceridemia y en las mujeres el HDL col bajo $(5,6)$, corroborado en Perú por Seclén en mestizos que viven a nivel del mar (19) y en nativos peruanos de altura (20). Sin embargo, en el presente trabajo, el componente HDL col bajo, no fue diferente entre mujeres NG y DG con SM (Gráfico 2). Se puede explicar considerando que el HDL global de todas las mujeres de la muestra en promedio es 45 $\mathrm{mg} / \mathrm{dl}$ (tabla no presentada), es decir, casi todas las NG y DG tienen HDL col bajo y sus edades oscilan entre 48-68 años (etapa menopáusica), que ratifica la alta prevalencia del SM y del HDL col bajo en este género y en edad menopáusica (21), que limita la diferencia entre mujeres NG y DG con SM.

También las diferencias encontradas podrían explicarse por el fenotipo metabólico de la población turca, cuya prevalencia menor de hipertrigliceridemia en pacientes con SM varía entre 35-71\% (22) en comparación al nuestro de 75\% (Tabla 2).

También en Turquía el 2015, al evaluar severidad del SM, el HDL col bajo resultó en un nivel sérico más bajo en los pacientes con 3 criterios de SM y el nivel sérico de TG fue mayor en los pacientes con SM con 5 criterios, aunque no se los dividió según normoglicemia o disglicemia y todos tenían sobrepeso u obesidad (23). Además, en China, el 2012, al evaluar riesgo de enfermedad arterial coronaria en pacientes con SM, el HDL col bajo fue el factor de riesgo común entre NG, pre diabéticos y diabéticos, sin diferencias significativas en su nivel sérico entre los grupos, pero si evidenciada con mayor nivel sérico de TG en los diabéticos con SM (24). Por lo que es importante en el análisis de todo trabajo con SM, la edad, sexo y el fenotipo poblacional, que determinará las diferencias proporcionales de sus componentes y de las subclases de SM.

Por fisiopatología el SM está muy unido a la RI $(1,25,26)$. Indirectamente tomando en cuenta el nivel de $\mathrm{TG}>130 \mathrm{mg} / \mathrm{dl}$ y $\mathrm{TG} / \mathrm{HDL}>3$ como los principales marcadores de RI (27). Encontramos que entre el $85-89 \%$ de los NG vs el $72-79 \%$ de los DG tuvieron RI con diferencia significativa (Tabla 1). Es decir, se encontró un mayor número de personas con $\mathrm{SM}$ y RI entre individuos NG que los DG; es por esta razón que la AACE (28), plantea que cuando aparece la diabetes mellitus tipo 2, se debería de dejar de hablar de RI o $\mathrm{SM}$, ya que pierden importancia, pues en esta secuencia metabólica disminuye la hiperinsulinemia, aparece la disglicemia y luego la diabetes mellitus tipo 2 .

Se postula que en la transición de un individuo normal, a un intolerante y luego aun diabético, la sensibilidad a la insulina se deteriora aproximadamente un $40 \%$, mientras la secreción de la insulina se deteriora 3 a 4 veces (29).

La deficiente inhibición de la lipasa sensible a hormonas por el estado de RI en los adipocitos conduce al aumento del flujo de ácidos grasos libres (AGL), que condiciona lipotoxicidad y es probable que juegue un rol en la progresión de tolerancia normal a la glucosa a hiperglicemia en ayunas (25). Los AGL generan inhibición de la lipoprotein lipasa tisular, generándose la dislipidemia aterogénica y el SM. También, estimulan a la célula beta la mayor producción de insulina, y así prosigue el círculo vicioso $(1,26,29)$.

Analizando los perfiles metabólicos de los sujetos con normoglicemia obesos, el Dr. Kissebach, propuso que tienen alterados el nivel de triglicéridos y HDL col $(30,31)$, que es el modelo que se objetiva en estudios como en Turquía (14) y en el presente trabajo; se plantea incluso que el metabolismo alterado de los AGL serían los causales de la RI (31), al competir con la glucosa por su captación periférica (32) además que incrementan sus niveles antes que la hiperglicemia se desarrolle $(32,33)$. Los AGL se unen a receptores de membrana como los TLR (34) (toll like receptors), y activan la cascada inflamatoria, forman ceramidas que bloquean la acción de la insulina al evitar la expresión los receptores GLUT 4 y menor captación celular de glucosa y mayor RI $(25,34,35)$. 
Proponemos que la disglicemia sería un estadio más avanzado del SM donde para algunos ya no debería considerarse como parte del síndrome de RI sobre todo cuando aparece la diabetes mellitus tipo 2 que es la posición de la $\operatorname{AACE}(3,28)$ ya que solo entre el 19 a $60 \%$ de diabéticos en este estadio, tienen hipertrigliceridemia $(36-38)$ a diferencia de las personas con SM que tienen entre el $70-90 \%$ (4-6,11,19); en el estado normo glicémico un buen marcador temprano serían los TG y no la glucosa (32) y además la hiperglicemia dentro de los estudios de prevalencia del SM es la de más baja prevalencia (4$6,11,15,22,24,39)$.

La principal limitación del presente estudio descriptivo, fue no tener mediciones directas de resistencia a la insulina y no haber podido separar con criterios estándares a los individuos del grupo disglicémicos según categorías de glucosa alterada en ayunas, intolerantes a la glucosa y/o diabéticos y poder diferenciar su perfil metabólico comparativamente con los normoglicémicos, muy importante de acuerdo a la secuencia metabólica que planteamos.

Como conclusiones encontramos que las diferencias de las frecuencias y mediciones estándar de los componentes del SM entre los pacientes con glucosa normal y disglicemia en ayunas en la población adulta de Trujillo, se da en el mayor nivel sérico de los TG en ayunas en el grupo NG y en la mayor frecuencia de hipertrigliceridemia en el grupo NG (en forma global, en hombres y mujeres) y también mayor frecuencia de HDL col bajo en el grupo de normoglicémicos (en forma global y en hombres).

\section{Declaración de financiamiento y de conflictos de intereses:}

El trabajo fue financiado por los autores, declaran no tener conflictos de intereses de parte de los autores

\section{Contribución de autoría:}

FJGM: diseño del estudio, análisis e interpretación de los resultados, elaboración del artículo, revisión crítica, aprobación de la versión final a ser publicada; JJHS: diseño del estudio, recolección de los datos, análisis e interpretación de los resultados, elaboración del artículo, revisión crítica, aprobación de la versión final a ser publicada; MAV: recolección de datos, análisis e interpretación de los resultados, revisión crítica; JGS: recolección de datos, análisis e interpretación de los resultados, elaboración del artículo, revisión crítica, elaboración de la versión final a ser publicada; JGA: recolección de datos, análisis e interpretación de los resultados, elaboración del artículo, revisión crítica, elaboración de la versión final a ser publicada; JOL: análisis e interpretación de los resultados, revisión crítica, elaboración del artículo.

\section{Correspondencia:}

Fernando Gross Melo, Av. Juan Pablo II 1110-B3. Trujillo, Perú Correo electrónico: f.gross@dr.com Teléfono 044949781248

\section{REFERENCIAS BIBLIOGRÁFICAS}

1. Rosas J, González A, Aschne P, et al. Consenso Latinoamericano de la Asociación Latinoamericana de Diabetes: Epidemiología, diagnóstico, control, prevención y tratamiento del Síndrome Metabólico en adultos. Rev ALAD. 2010; 18:25-44.

2. Alberti KGMM, Eckel RH, Grundy SM, et al. Harmonizing the metabolic syndrome: a joint interim statement of the International Diabetes Federation Task Force on Epidemiology and Prevention; National Heart, Lung, and Blood Institute; American Heart Association; World Heart Federation; International Atherosclerosis Society; and International Association for the Study of Obesity. Circulation. 2009; 120(16):1640-5.

3. Sperling LS, Mechanick JI, Neeland IJ, et al. The CardioMetabolic Health Alliance: Working Toward a New Care Model for the Metabolic Syndrome. J Am Coll Cardiol. 2015; 66(9):1050-67.

4. Pajuelo J, Sánchez J. El síndrome metabólico en adultos, en el Perú. An Fac med. 2007; 68(1): 38-46. (Fecha de acceso 15 de julio del 2018) Disponible en: http://www.scielo.org.pe/scielo.php?script=sci_artte xt\&pid=S1025-55832007000100005

5. Aliaga E, Tello T, Varela L, Seclén S, Ortiz P, Chávez H. Frecuencia de síndrome metabólico en adultos mayores del Distrito de San Martin de Porres de Lima, Perú según los criterios de ATP III y de la IDF. Rev Med Hered. 2014; 25(3):142-8.

6. Galarreta A, Donet M, Huamán J. Síndrome metabólico en la población adulta de Trujillo de acuerdo a diferentes definiciones. Acta Médica Peru. 2009; 26(4):217-25.

7. Kahn R, Buse J, Ferrannini E, Stern M, American Diabetes Association, European Association for the Study of Diabetes. The metabolic syndrome: time for a critical appraisal: joint statement from the American Diabetes Association and the European Association for the Study of Diabetes. Diabetes Care. 2005; 28(9):2289-304. 
8. Garber AJ, Handelsman Y, Einhorn D, et al. Diagnosis and management of prediabetes in the continuum of hyperglycemia: when do the risks of diabetes begin? A consensus statement from the American College of Endocrinology and the American Association of Clinical Endocrinologists. Prediabetes Consensus Statement, Endocr Pract. 2008; 14(7):93346.

9. García GM, Madrid GS, Diaz MR, et al. Concordancia entre cinco definiciones de síndrome metabólico: Cartagena, Colombia. Rev Esp Salud Pública. 2012; 86(3):301-311.

10. Cárdenas H, Sánchez J, Roldán L, Mendoza TF. Prevalence of metabolic syndrome in people 20 years old and more. Peru, 2005. Rev Esp Salud Pública. 2009; 83(2):257-65.

11. Castillo K, Ríos M, Huaman J. Frecuencia y características de la glicemia basal alterada en adultos de Trujillo según criterios diagnósticos. Acta Médica Peru. 2011; 28(3):132-45.

12. Thomas GN, McGhee SM, Schooling CM, et al. Determinants of normoglycemia and contribution to cardiovascular risk factors in a Chinese population: the Hong Kong Cardiovascular Risk Factor Study. J Endocrinol Invest. 2006; 29(6):528-35.

13. Hsiao F-C, Hsieh C-H, Wu C-Z, et al. Elevated fasting glucose levels within normal range are associated with an increased risk of metabolic syndrome in older women. Eur J Intern Med. 2013; 24(5):425-9.

14. Uzunlulu M, Oguz A. Is metabolic syndrome a condition independent of prediabetes and type 2 diabetes mellitus? A report from Turkey. Endocr J. 2007; 54(5):745-50.

15. Linares-Reyes E, Castillo-Minaya K, Ríos-Mino M, Huamán-Saavedra J. Estudio de correlación entre los diagnósticos de cintura hipertrigliceridémica y síndrome metabólico en adultos de Trujillo, Perú. Rev Peru Med Exp Salud Publica. 2014; 31(2):254260.

16. American Diabetes Association. 2. Classification and Diagnosis of Diabetes: Standards of Medical Care in Diabetes-2018. Diabetes Care. 2018; 41(Suppl 1):S13-27.

17. Deepa M, Papita M, Nazir A, et al. Lean people with dysglycemia have a worse metabolic profile than centrally obese people without dysglycemia. Diabetes Technol Ther. 2014; 16(2):91-6.

18. Osada J, Vidal L, León F. Comparación de variables: Clavando un tornillo. Rev Médica Chile. 2014; 142(8): 1080-1081. (Fecha de acceso 15 de julio del 2018) Disponible en: http://revistamedicadechile.cl/ ojs/index.php/rmedica/article/view/3520

19. Seclén S, Villena A, Larrad MT, et al. Prevalence of the metabolic syndrome in the mestizo population of peru. Metab Syndr Relat Disord. 2006; 4(1):1-6.

20. Mohanna S, Baracco R, Seclén S. Lipid profile, waist circumference, and body mass index in a high altitude population. High Alt Med Biol. 2006; 7(3):245-55.

21. Rojas S, Lopera J, Cardona J, Vargas N, Hormaza M. Síndrome metabólico en la menopausia, conceptos clave. Rev Chil Obstet Ginecol. 2014; 79(2):121-8.

22. Gündogan K, Bayram F, Capak M, et al. Prevalence of metabolic syndrome in the Mediterranean region of Turkey: evaluation of hypertension, diabetes mellitus, obesity, and dyslipidemia. Metab Syndr Relat Disord. 2009; 7(5):427-34.

23. Turker Y, Baltaci D, Turker Y, et al. Investigation of relationship of visceral body fat and inflammatory markers with metabolic syndrome and its components among apparently healthy individuals. Int J Clin Exp Med. 2015; 8(8):13067-77.

24. Gong X, Pan X, Chen X, Hong C, Hong J, Shen F. Associations between coronary heart disease and individual components of the metabolic syndrome according to glucose tolerance status. J Int Med Res. 2012; 40(3):934-42.

25. Luna-Luna M, Medina-Urrutia A, Vargas-Alarcón G, Coss-Rovirosa F, Vargas-Barrón J, Pérez-Méndez Ó. Adipose tissue in metabolic syndrome: Onset and progression of atherosclerosis. Arch Med Res. 2015; 46(5):392-407.

26. Haffner SM, Valdez RA, Hazuda HP, Mitchell BD, Morales PA, Stern MP. Prospective analysis of the insulin-resistance syndrome (syndrome $\mathrm{X}$ ). Diabetes. 1992; 41(6):715-22.

27. McLaughlin T, Abbasi F, Cheal K, Chu J, Lamendola C, Reaven G. Use of metabolic markers to identify overweight individuals who are insulin resistant. Ann Intern Med. 2003; 139(10):802-9.

28. Einhorn D, Reaven GM, Cobin RH, et al. American College of Endocrinology position statement on the insulin resistance syndrome. Endocr Pract Off J Am Coll Endocrinol Am Assoc Clin Endocrinol. 2003; 9(3):237-52.

29. Groop L. Pathogenesis of type 2 diabetes: the relative contribution of insulin resistance and impaired insulin secretion. Int J Clin Pract Suppl. 2000; (113):3-13.

30. Olivier M. Body fat distribution, lipoprotein metabolism, and insulin resistance: a lifetime of research on the pathophysiology of the human metabolic syndrome. J Clin Lipidol. 2012; 6(6):6013.

31. Castro M, de Bruin TW, de Valk HW, Shoulders CC, Jansen H, Willem Erkelens D. Impaired fatty acid metabolism in familial combined hyperlipidemia.

32. A mechanism associating hepatic apolipoprotein B overproduction and insulin resistance. J Clin Invest. 1993; 92(1):160-8.

33. Ferrannini E, Barrett EJ, Bevilacqua S, DeFronzo RA. Effect of fatty acids on glucose production and utilization in man. J Clin Invest. 1983; 72(5):173747. 
34. Queiroz JCF de, Alonso-Vale MIC, Curi R, Lima FB. Control of adipogenesis by fatty acids. Arq Bras Endocrinol Amp Metabol. 2009; 53(5):582-94.

35.3Shi H, Kokoeva MV, Inouye K, Tzameli I, Yin $\mathrm{H}$, Flier JS. TLR4 links innate immunity and fatty acid-induced insulin resistance. J Clin Invest. 2006; 116(11):3015-25.

36. Carrasco F, Galgani J , Reyes M. Síndrome de resistencia a la insulina. estudio y manejo. Revista Médica Clínica Las Condes. 2013; 24(5): 827-837. doi: https://doi.org/10.1016/S0716-8640(13)70230-X

37. McGarry JD. Banting lecture 2001: dysregulation of fatty acid metabolism in the etiology of type 2 diabetes. Diabetes. 2002; 51(1):7-18.
38. Carmena R. Riesgo elevado de disfunción lipoproteica en la diabetes mellitus tipo 2. Rev Esp Cardiol Supl. 2008; 8(3):19C-26C.

39. Mooradian AD. Dyslipidemia in type 2 diabetes mellitus. Nat Clin Pract Endocrinol Metab. 2009; 5(3):150-9.

40. Panazzolo DG, Sicuro FL, Clapauch R, Maranhão PA, Bouskela E, Kraemer-Aguiar LG. Obesity, metabolic syndrome, impaired fasting glucose, and microvascular dysfunction: a principal component analysis approach. BMC Cardiovasc Disord. 2012; 12:102.

Recibido: $22 / 11 / 2017$

Aceptado: 10/06/2018 